\title{
Null syndrome
}

INSERM

\section{Source}

INSERM. (1999). Orphanet: an online rare disease and orphan drug data base. Null syndrome. ORPHA:280234

The null syndrome is part of the Pelizaeus-Merzbacher disease (PMD; see this term) spectrum and is characterized by mild PMD features associated with demyelinating peripheral neuropathy. 